\title{
Prostate stem cell antigen is expressed in normal and malignant human brain tissues
}

\author{
HIROE ONO, HIROMI SAKAMOTO, TERUHIKO YOSHIDA and NORIHISA SAEKI \\ Division of Genetics, National Cancer Center Research Institute, Tokyo 104-0045, Japan
}

Received June 25, 2015; Accepted October 24, 2016

DOI: $10.3892 / \mathrm{ol} .2017 .7632$

\begin{abstract}
Prostate stem cell antigen (PSCA) is a glycosylphosphatidylinositol (GPI)-anchored cell surface protein and exhibits an organ-dependent expression pattern in cancer. PSCA is upregulated in prostate cancer and downregulated in gastric cancer. PSCA is expressed in a variety of human organs. Although certain studies previously demonstrated its expression in the mammalian and avian brain, its expression in the human brain has not been thoroughly elucidated. Additionally, it was previously reported that PSCA is weakly expressed in the astrocytes of the normal human brain but aberrantly expressed in glioma, suggesting that PSCA is a promising target of glioma therapy and prostate cancer therapy. The current study identified PSCA expression in the neural and choroid plexus cells of the normal human brain by immunohistochemistry. In brain tumors, PSCA was expressed in medulloblastoma and glioma, and its expression was also observed in papilloma and papillary carcinoma of the choroid plexus, ependymoma and meningioma. The results suggest that PSCA may have a tumor-promoting function in brain tumors and is a potential target for their therapy. However, its expression in normal neuronal and choroid plexus cells implies that a PSCA-targeted therapy may lead to certain adverse phenomena.
\end{abstract}

\section{Introduction}

The prostate stem cell antigen (PSCA) gene encodes a glycosylphosphatidylinositol (GPI)-anchored membrane protein (1). Although its biological function is unknown, it is thought that PSCA is localized to lipid rafts on the outer surface of the cell membrane, a special microdomain enriched in glycosphingolipids, cholesterol and other lipidated proteins, and has certain

Correspondence to: Dr Norihisa Saeki, Division of Genetics, National Cancer Center Research Institute, 5-1-1 Tsukiji, Chuo-ku, Tokyo 104-0045, Japan

E-mail: nsaeki@ncc.go.jp

\section{Abbreviations: PSCA, prostate stem cell antigen}

Key words: prostate stem cell antigen, GPI-anchored protein, immunohistochemistry, neural cells, brain tumor functions in subcellular signal transduction (2). Originally, PSCA was identified as a gene upregulated in prostate cancer (3), and later its upregulation was demonstrated in other types of tumor, including urinary bladder cancer, renal cell carcinoma, hydatidiform mole and ovarian mucinous tumor, where PSCA is thought to be involved in tumor progression (1). However, downregulation of the gene was reported in gastric and gallbladder cancer, where it may act as a tumor suppressor $(4,5)$. As another pattern of its expression in cancer, PSCA is not expressed in the ductal cells of the normal pancreas or the epithelial cells in the normal lung, however, it is expressed in their malignant counterparts, pancreatic cancer and non-small cell lung cancer $(6,7)$.

It was previously reported that PSCA expression was almost absent in the normal human brain but was detected in glioma, and it may represent a suitable target for immunotherapy of gliomas (8). Additionally, PSCA protein was recently detected in human brain cortex by western blot analyses (9). PSCA expression in the brain was also reported in other animals, including in the cerebellum, telencephalon and autonomic ganglia of chick embryos and adult mice, and in the frontal cortex and hippocampus of rats $(10,11)$. However, the detail of its expression in the human brain is yet to be elucidated.

The current study aimed to examine PSCA expression sites in the normal human brain. Immunohistochemistry was performed using a mouse monoclonal anti-PSCA antibody, which revealed its expression in several types of normal cells in the brain. The expression status in their malignant counterparts was also examined.

\section{Materials and methods}

Reverse transcription-quantitative polymerase chain reaction (RT-qPCR). Total RNA samples isolated from normal human brain tissues were obtained from Clontech Laboratories, Inc. (Mountain View, CA, USA), which include frontal lobe (cat. no. 636563), temporal lobe (cat. no. 636564), parietal lobe (cat. no. 636571), occipital lobe (cat. no. 636570), paracentral gyrus (cat.no. 636574), postcentral gyrus (cat. no. 636573), insula (cat. no. 636568), corpus callosum (cat. no. 636567), putamen (cat. no. 636575), substantia nigra (cat. no. 636560), nucleus ambiguous (cat. no. 636569), hippocampus (cat. no. 636593), cerebellum (cat. no. 636535), pons (cat. no. 636572), medulla oblongata (cat. no. 636562) and stomach RNA (BioChain Institute Inc., Newark, CA, USA). The templates cDNA were synthesized using the ThermoScript RT-PCR system 
Table I. Results of immunohistochemical analysis of prostate stem cell antigen expression in brain tumors.

\begin{tabular}{lrcc}
\hline Cancer type & Tumor & Cases & Expression $(\%)^{\mathrm{a}}$ \\
\hline Medulloblastoma & 14 & 14 & 100.0 \\
Astrocytoma & & & \\
I & 16 & 12 & 75.0 \\
II & 49 & 41 & 83.7 \\
III & 6 & 6 & 100.0 \\
IV & 9 & 9 & 100.0 \\
Oligodendroglioma & & & \\
II & 6 & 5 & 83.3 \\
III & 2 & 2 & \\
Ependymoma & & & 100.0 \\
II & 3 & 3 & 100.0 \\
III & 3 & 3 & \\
Ependymoblastoma & & & \\
IV & 2 & 2 & 100.0 \\
Choroid plexus & & & \\
Papilloma & 2 & 2 & \\
Papillary carcinoma & 1 & 1 & \\
Meningioma & & & \\
Meningotheliomatous & 3 & 3 & \\
Fibrous & 15 & 15 & \\
Transitional & 10 & 10 & \\
\hline
\end{tabular}

aTumor types with less than three cases are excluded.

(Invitrogen; Thermo Fisher Scientific, Inc., Waltham, MA, USA). qPCR was performed using a TaqMan Gene Expression assay (assay ID Hs00194665_m1 for PSCA; Applied Biosystems; Thermo Fisher Scientific, Inc.; cat. no. 4326317E for GAPDH), which was conducted for 40 cycles of $95^{\circ} \mathrm{C}$ for $15 \mathrm{sec}$ and $60^{\circ} \mathrm{C}$ for $60 \mathrm{sec}$, using ABI PRISM 7900HT Sequence Detection system (Thermo Fisher Scientific, Inc.). The relative transcript level was calculated using the $2^{-\Delta \Delta \mathrm{Cq}}$ method (12) with $G A P D H$ transcript as a reference, as it had been demonstrated in our previous studies that $G A P D H$ is an excellent reference for quantification of the PSCA expression (4,5). For gel electrophoresis, RT-PCR was conducted for 35 cycles of $94^{\circ} \mathrm{C}$ for $60 \mathrm{sec}$, 5894 for $60 \mathrm{sec}$ and $60^{\circ} \mathrm{C}$ for $60 \mathrm{sec}$ using the following primer pairs: PSCA, 5'-TGGAGAACTGCACCCAGCT-3' and 5'-GAC TTGCGTTAGGATGTGCC-3'; ACTB, 5'-TCATCACCATTG GCAATGAG-3' and 5'-CACTGTGTTGGCGTACAGGT-3'.

Immunohistochemistry. Embedded normal brain tissue specimens on glass were purchased from BioChain Institute, Inc. (cat. nos. T8234564, T8234444, T8234445, T2234043 and T2234044). The PSCA expression in brain tumor tissues was examined using two tissue arrays: Brain disease spectrum (brain cancer progression) tissue array (cat. no. CNS801; US Biomax, Inc., Rockville, MD, USA), and multiple brain cancer and normal adjacent tissue array (cat. no. GL1001; US Biomax, Inc.). Immunohistochemistry was performed with mouse monoclonal anti-PSCA antibod, which was produced

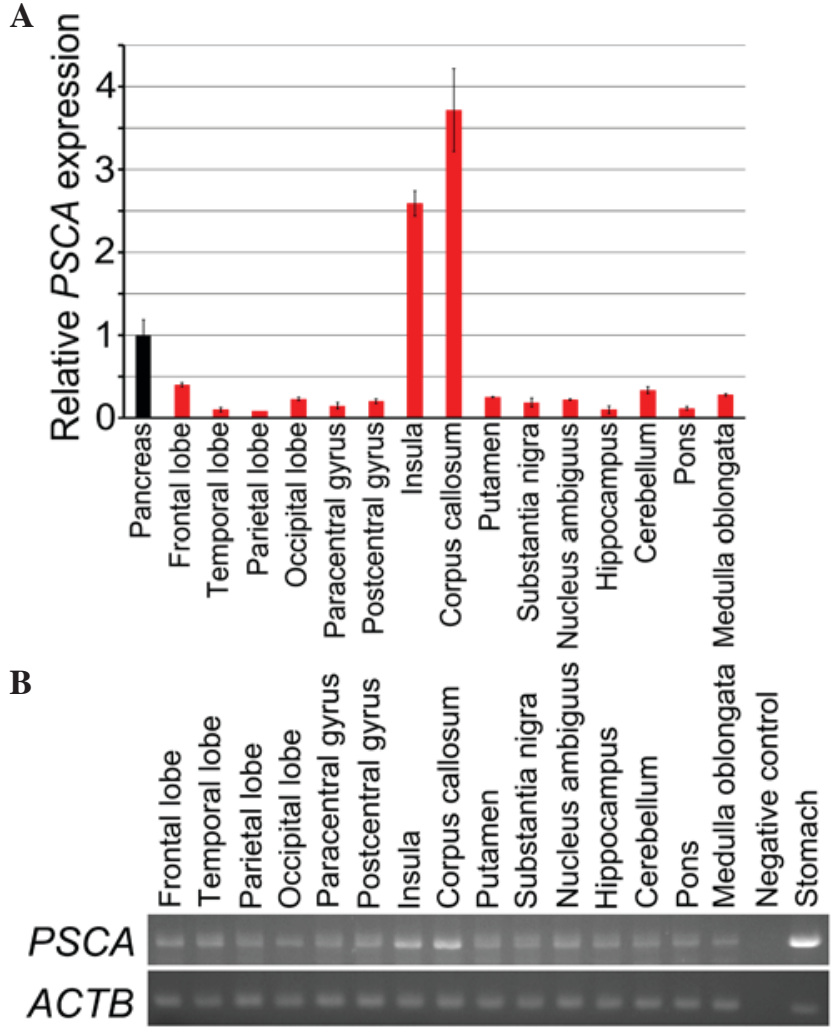

Figure 1. PSCA expression in human brain. (A) RT-qPCR detected PSCA transcripts in RNA samples from normal human brain. The amount of the transcript in pancreatic RNA, not in stomach RNA, was used as the reference, as it was demonstrated that stomach expresses a higher amount of the transcript than the brain tissues. The $\mathrm{Cq}$ value for the negative control (PCR product with water as the template) was not obtained. Values are presented as the mean \pm standard deviation. (B) The RT-PCR result demonstrated by gel electrophoresis. PSCA, prostate stem cell antigen; ACTB, $\beta$-actin. RT-qPCR, reverse transcription-quantitative polymerase chain reaction.

in our previous study (4) and whose specificity had been demonstrated previously $(4,5)$, and rabbit anti-PCNA antibody (cat. no. sc-7907; Santa Cruz Biotechnology, Dallas, Texas, USA). The sections were incubated at $4^{\circ} \mathrm{C}$ overnight with both antibodies (each 1:50 dilution) simultaneously, and then with an alkaline phosphatase-conjugated anti-mouse $\mathrm{IgG}$ antibody (cat. no. 414241; NICHIREI Biosciences, Inc., Tokyo, Japan) and peroxidase-conjugated anti-rabbit IgG antibody (cat. no. 424141; NICHIREI Biosciences, Inc.), at original concentration, for $30 \mathrm{~min}$ at room temperature. PSCA protein was visualized using Alkaline Phosphatase Substrate kit III (Vector Laboratories, Inc., Burlingame, CA, USA) and PCNA by VECTOR NovaRED Substrate kit for Peroxidase (Vector Laboratories, Inc.). For immunocytochemistry, using SuperFect Transfection Reagent (Qiagen, Inc., Valencia, CA, USA), a pcDNA3.1 construct for expressing PSCA sense strand or that expressing PSCA antisense was introduced into HSC-57 gastric cancer cell line, not into brain tissue-derived cell lines, as it is the cell line in which no PSCA expression had been demonstrated in our previous studies $(4,5)$. HSC-57 was established and provided by Dr. Kazuyoshi Yanagihara (4) and maintained in Dulbecco's modified Eagle's medium supplemented with $10 \%$ bovine serum at $37^{\circ} \mathrm{C}$ under an atmosphere of $5 \% \mathrm{CO}_{2}$. The immunocytochemistry was performed in the 


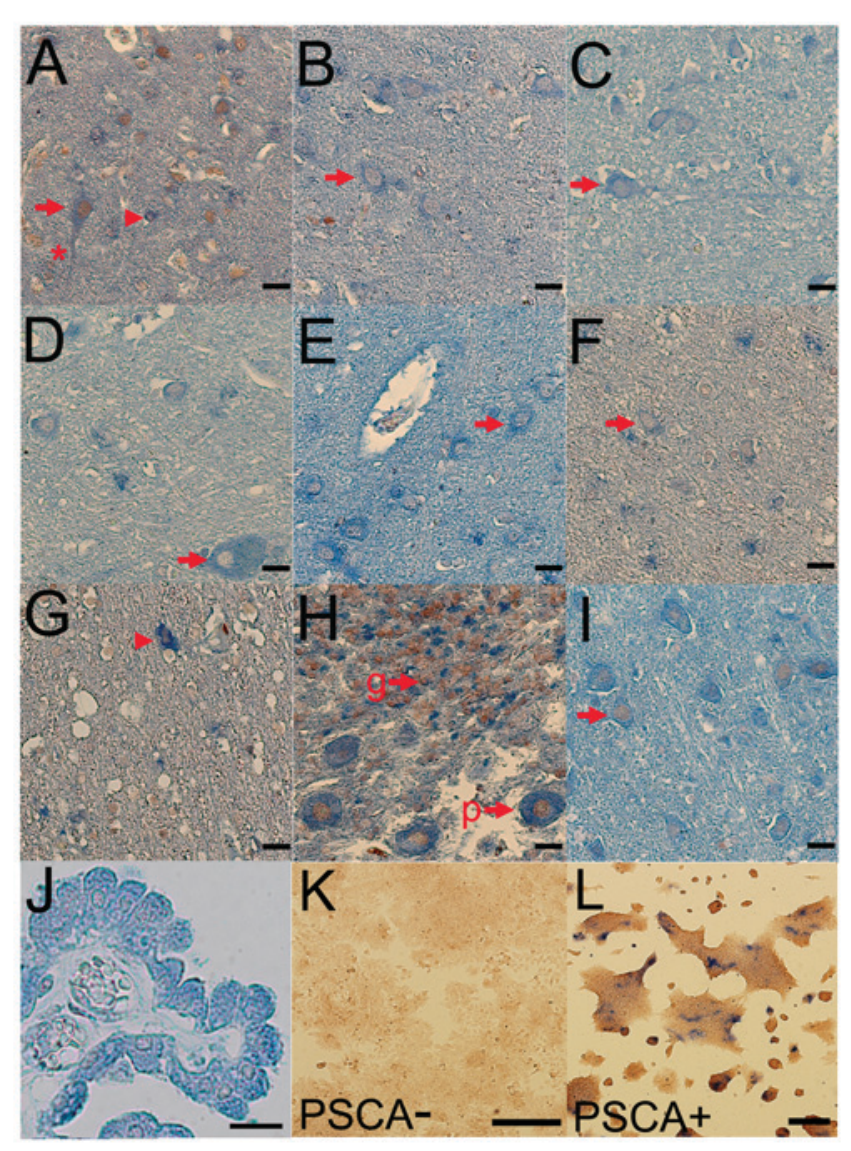

Figure 2. PSCA expression in human brain. Immunohistochemistry, double staining with anti-PSCA (blue) and anti-proliferating cell nuclear (PCNA, brown) antibodies, revealed weak PSCA expression in the neural and glial cells in the human brain tissues. The arrows indicate staining in the perikaryon of the neuron. (A) Frontal lobe cortex; the asterisk indicates staining in the dendrite of the neuron. (B) Precentral gyrus cortex. (C) Postcentral gyrus cortex. (D) Temporal lobe. (E) Parietal lobe. (F) Occipital lobe. (G) Corpus callosum; blue-stained cell with small nucleus, indicated by arrowhead, is the glial cell. (H) Celleberum; p, Purkinje cell. g, granule cell. (I) Pons. (J) Choroid plexus. A gastric cancer cell line (K) with and (L) without expressing PSCA protein. Scale bars, $20 \mu \mathrm{m}$. PSCA, prostate stem cell antigen.

same manner as the immunohistochemistry, after fixing the cells on the chamber slides using $4 \%$ paraformaldehyde.

\section{Results}

PSCA is expressed in neural and choroid plexus cells in the normal human brain. Initially, PSCA expression in normal human brain tissues was examined by RT-qPCR (Fig. 1A). PSCA transcripts were detected in all the tissues examined, however the expression level was weak; the mean $\Delta \mathrm{Cq}$ values of the samples were $<30$, except in the insula and corpus callosum, exhibiting a far lower expression compared with the level in the stomach (data not shown). Consequently, the amount of the PSCA transcripts in each tissue is demonstrated in Fig. 1A, in comparison with the amount in the pancreas, which expresses PSCA at a lower level than the stomach (13). However, analyses of a standard curve for PSCA amplification revealed that the expression level of the tissue was so low that it may be out of the range in which the $\mathrm{Cq}$ values precisely reflect the difference in the amount of the transcripts among the tissues. Consequently, the result may be lacking in

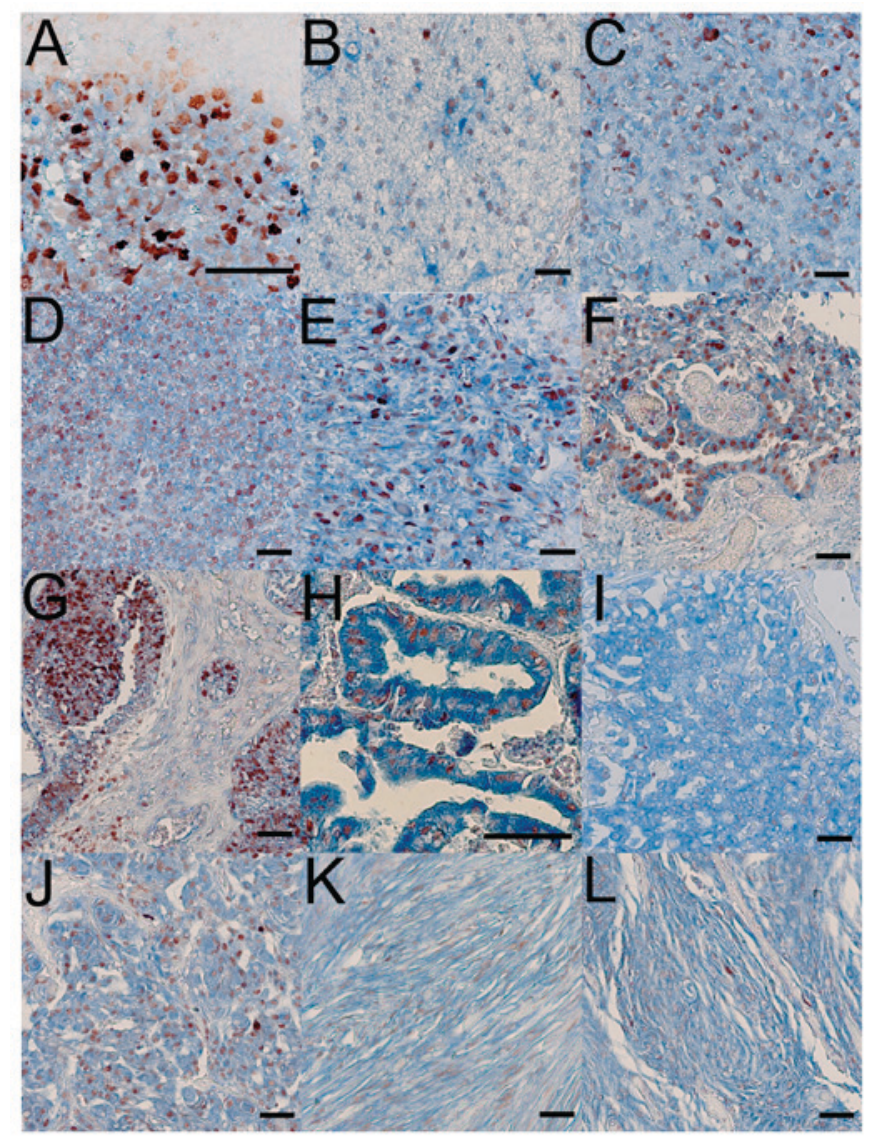

Figure 3. PSCA is expressed in brain tumors. Immunohistochemistry, double staining with anti-PSCA (blue) and anti-proliferating cell nuclear (brown) antibodies, exhibits PSCA expression in the brain tumor cells: (A) medulloblastoma; (B) astrocytoma grade II; (C) astrocytoma grade III; (D) astrocytoma grade IV; (E) oligodendroglioma grade II; (F) ependymoma; (G) ependymoblastoma; (H) choroid plexus papilloma; (I) choroid plexus papillary carcinoma; (J) meningotheliomatous meningioma; (K) fibrous meningioma and (L) transitional meningioma. Scale bars, $50 \mu \mathrm{m}$. PSCA, prostate stem cell antigen.

precise quantitativity. Amplified product was also observed for all the samples by agarose gel electrophoresis (Fig. 1B).

Subsequently, double-staining immunohistochemistry was performed for PSCA and PCNA (proliferating cell nuclear antigen), which detected PSCA proteins in the frontal lobe (Fig. 2A), precentral gyrus (Fig. 2B), postcentral gyrus (Fig. 2C), temporal lobe (Fig. 2D), parietal lobe (Fig. 2E), occipital lobe (Fig. 2F), corpus callosum (Fig. 2G), celleberum (Fig. 2H) and pons (Fig. 2I) of the normal human brain (Fig. 2). The PSCA staining was predominantly observed in the perikaryon, a cell body consisting of a nucleus and the surrounding cytoplasm, but also in the dendrites of neuronal cells (asterisk in Fig. 2A). The expression level was weak, compared with that in gastric and gallbladder epithelium $(4,5)$, which reduced the signal/noise ratio in this immunohistochemical expression analysis. In addition to the neuronal cells, PSCA expression was also detected in choroid plexus cells (Fig. 2J). PSCA dependent staining was confirmed by imunocytochemistry using a gastric cancer cell line with and without PSCA expression (Fig. 2K and L).

PSCA is expressed in brain tumors. As PSCA exhibits an organ-dependent expression pattern in cancer (1), its expression status was investigated in brain tumors using 
immunohistochemistry (Fig. 3 and Table I). The current study detected PSCA expression in medulloblastoma (Fig. 3A), and, in 8 of 14 cases, the staining was clearly stronger than in normal neuronal cells in the cerebral cortex.

As previously demonstrated in another study (8),PSCA expression was observed in glioma, including astrocytoma (Fig. 3B-D) and oligodedroglioma (Fig. 3E), and its positive staining rate in astrocytoma is associated with stage-progression; $75 \%$ in grade I, $83.7 \%$ in grade II, $100 \%$ in grade III and IV. In addition to glioma, PSCA expression was also exhibited in ependymoma (Fig. 3F) and ependymoblastoma (Fig. 3G), and a relatively strong PSCA expression was revealed in choroid plexus papilloma (Fig. 3H) and papillary carcinoma (Fig. 3I), although this seems to reflect the expression status in normal choroid plexus cells (Fig. 2J).

Furthermore, PSCA expression was observed in meningioma (Fig. 3J-L). It appeared to be upregulated in all the specimens, as PSCA expression was not detected in the normal meninges in the current immunohistochemistry analysis (data not shown). However, the relative level of the PSCA signal in meningioma compared with in normal meninges was not evaluated because the tissue array contained no reference for meningeal expression.

\section{Discussion}

The current study demonstrated PSCA expression in the neuronal cells of the brain, to the best of our knowledge, for the first time. However, its function in human brain and the brain of other animals is unknown. Although unknown within the central nervous system, the function of PSCA in neuronal cells is well elucidated in the peripheral ganglion of the chick. In chick ciliary ganglion, $\alpha 7$-containing nicotinic acetylcholine receptors have a role in the induction of the developmentally regulated cell death of neuronal cells, and PSCA has an antagonistic function against the cell death induction and rescues neuronal cells from cell death (10). In the development of the ganglion, PSCA expression is also developmentally regulated. A previous study reported developmental regulation of PSCA expression in the rat brain, however, in the study, the expression was examined by RT-PCR analyses on brain tissues, not by immunohistochemistry to reveal the precise cell types with PSCA expression (11). Notably, PSCA knockout mice were viable and exhibited no gross abnormality (14).

The PSCA expression in the choroid plexus is notable, as it is a type of ependymal cells specialized for cerebrospinal fluid production. PSCA is thought to have a role in cell differentiation and proliferation in certain epithelial tissues, including gastric epithelium. However, its expression in gallbladder epithelium, which consists of a single layer of the cells and functions in absorbing water and electrolytes for bile condensation, suggests that it may have a simple function in the gallbladder. PSCA may also have a simple function, such as fluid import and export in the choroid plexus.

To the best of our knowledge, the current study is the first to demonstrated that PSCA is expressed in medulloblastoma and meningioma. As reported in a previous study (8), the PSCA expression status is positively correlated to the grade of astrocytoma (Table I).

The results of the current study suggest that, in the brain tumors, PSCA has a tumor-promoting function and may be a promising therapeutic target. However, it is also important to note that PSCA is expressed in the normal brain, because it is considered as a target molecule in for treatment of pancreatic and prostate cancers, in addition to brain tumors $(15,16)$. Thus, clinical applications targeting PSCA have a potential risk of leading to adverse events in the central nervous system.

\section{Acknowledgements}

This study was supported by a JST grant for the personalized medicine project and by JSPS KAKENHI (grant no. 23501327).

\section{References}

1. Saeki N, Gu J, Yoshida T and Wu X: Prostate stem cell antigen: A Jekyll and Hyde molecule? Clin Cancer Res 16: 3533-3538, 2010.

2. Sharom FJ and Radeva G: GPI-anchored protein cleavage in the regulation of transmembrane signals. Subcell Biochem 37: 285-315, 2004.

3. Reiter RE, Gu Z, Watabe T, Thomas G, Szigeti K, Davis E, Wahl M, Nisitani S, Yamashiro J, Le Beau MM, et al: Prostate stem cell antigen: A cell surface marker overexpressed in prostate cancer. Proc Natl Acad Sci USA 95: 1735-1740, 1998.

4. Study Group of Millennium Genome Project for Cancer; Sakamoto H, Yoshimura K, Saeki N, Katai H, Shimoda T, Matsuno Y, Saito D, Sugimura H, Tanioka F, et al: Genetic variation in PSCA is associated with susceptibility to diffuse-type gastric cancer. Nat Genet 40: 730-740, 2008.

5. Ono H, Hiraoka N, Lee YS, Woo SM, Lee WJ, Choi IJ, Saito A Yanagihara K, Kanai Y, Ohnami S, et al: Prostate stem cell antigen, a presumable organ-dependent tumor suppressor gene, is down-regulated in gallbladder carcinogenesis. Genes Chromosomes Cancer 51: 30-41, 2012.

6. Argani P, Rosty C, Reiter RE, Wilentz RE, Murugesan SR, Leach SD, Ryu B, Skinner HG, Goggins M, Jaffee EM, et al: Discovery of new markers of cancer through serial analysis of gene expression: Prostate stem cell antigen is overexpressed in pancreatic adenocarcinoma. Cancer Res 61: 4320-4324, 2001.

7. Kawaguchi T, Sho M, Tojo T, Yamato I, Nomi T, Hotta K, Hamada K, Suzaki Y, Sugiura S, Kushibe K, et al: Clinical significance of prostate stem cell antigen expression in non-small cell lung cancer. Jpn J Clin Oncol 40: 319-326, 2010.

8. Geiger KD, Hendruschk S, Rieber EP, Morgenroth A, Weigle B, Juratli T, Senner V, Schackert G and Temme A: The prostate stem cell antigen represents a novel glioma-associated antigen. Oncol Rep 26: 13-21, 2011.

9. Jensen MM, Arvaniti M, Mikkelsen JD, Michalski D, Pinborg LH, Härtig W and Thomsen MS: Prostate stem cell antigen interacts with nicotinic acetylcholine receptors and is affected in Alzheimer's disease. Neurobiol Ageing 36: 1629-1638, 2015.

10. Hruska M, Keefe J, Wert D, Tekinay AB, Hulce JJ, Ibañez-Tallon I and Nishi R: Prostate stem cell antigen is an endogenous lynx1-like prototoxin that antagonizes alpha7-containing nicotinic receptors and prevents programmed cell death of parasympathetic neurons. J Neurosci 29: 14847-14854, 2009.

11. Thomsen MS, Cinar B, Jensen MM, Lyukmanova EN, Shulepko MA, Tsetlin V, Klein AB and Mikkelsen JD: Expression of the Ly-6 family proteins Lynx 1 and Ly6H in the rat brain is compartmentalized, cell-type specific, and developmentally regulated. Brain Struct Funct 219: 1923-1934, 2014.

12. Livak KJ and Schmittgen TD: Analysis of relative gene expression data using real-time quantitative PCR and the 2- $\Delta \Delta \mathrm{CT}$ method. Methods 25: 402-408, 2001.

13. Ono H, Yanagihara K, Sakamoto H, Yoshida T and Saeki N. Prostate stem cell gene is expressed in islets of pancreas. Anat Cell Biol 45: 149-154. 2012.

14. Moore ML, Teitell MA, Kim Y, Watabe T, Reiter RE, Witte ON and Dubey P: Deletion of PSCA increases metastasis of TRAMP-induced prostate tumors without altering primary tumor formation. Prostate 68: 139-151, 2008.

15. Wente MN, Jain A, Kono E, Berberat PO, Giese T, Reber HA, Friess H, Büchler MW, Reiter RE and Hines OJ: Prostate stem cell antigen is a putative target for immunotherapy in pancreatic cancer. Pancreas 31: 119-125, 2005.

16. Morris MJ, Eisenberger MA, Pili R, Denmeade SR, Rathkopf D, Slovin SF, Farrelly J, Chudow JJ, Vincent M, Scher HI and Carducci MA: A phase I/IIA study of AGS-PSCA for castration-resistant prostate cancer. Ann Oncol 23: 2714-2719, 2012. 